\title{
Cross-Sectional survey to ascertain the prevalence of Molar Incisor Hypo-mineralization in the Trinidad and Tobago population
}

\author{
Trudee Hoyte ${ }^{1 *}$, Anne Kowlessar ${ }^{2}$, Anil Ali ${ }^{3}$ and David Bearn ${ }^{4}$ \\ ${ }^{1}$ Department of Orthodontics, University of The West Indies, St. Augustine, Trinidad \\ ${ }^{2}$ Department of Pediatric Dentistry, University of The West Indies, St. Augustine, Trinidad \\ ${ }^{3}$ Department of Orthodontics, University of The West Indies, St. Augustine, Trinidad \\ ${ }^{4}$ Department of Orthodontics, University of Dundee, UK
}

*Corresponding author: Trudee Hoyte, School of Dentistry, University of The West Indies, St. Augustine, Trinidad.

To Cite This Article: Trudee Hoyte, Cross-Sectional survey to ascertain the prevalence of Molar Incisor Hypo-mineralization in the Trinidad and Tobago population. 2020 - 7(3). AJBSR.MS.ID.001141. DOI: 10.34297/AJBSR.2020.07.001141.

Received: 眥 January 30, 2020; Published: 眥 February 11, 2020

Abstract

Aim: To determine the prevalence of molar incisor hypomineralization (MIH) in 11 to 12-year olds in the Trinidad and Tobago population and to determine if there is any association with ethnicity and gender.

Design: Cross-sectional survey.

Materials and Methods: First permanent molars and all permanent incisors were examined in 532 children comprising 317 females (58.1\%) and 229 males (41.9\%). The sample were 11-12 years olds (mean age 11.9 years) in high schools across Trinidad and Tobago. Examinations were conducted by one examiner.

Results: Afro-Trinidadian subjects comprised $62 \%$ of the sample, Indo- Trinidadian subjects $22 \%$ and Mixed ethnicity $16 \% .7$ out of 532 subjects had at least one molar with hypomineralization which represents overall prevalence of $1.3 \%$. Maxillary molars were more frequently hypomineralized and females showed a higher prevalence than males.

Conclusion: The prevalence of molar incisor hypomineralization in Trinidad and Tobago population was 1.3\% in the 11-12 ears age group.

Keywords: Cross-sectional survey; Molar Incisor Hypomineralization; Prevalence, Trinidad and Tobago

\section{Introduction}

Molar incisor hypomineralization (MIH) is a developmental defect affecting the molars and incisors. It is defined as hypomineralization of systemic origin of one to four permanent first molars frequently associated with affected incisors and these molars are related to major clinical problems in severe cases [1]. The colour of the affected enamel is white, whitish-yellow or yellowish brown and the defect has usually a clear border between affected and sound enamel [2].Other names include idiopathic hypomineralization in first permanent molars, non-fluoride hypomineralization, cheese molar [3], condition hypomineralized first permanent molar [4]. A etiological associations with systemic conditions or environmental insults during the child's first 3 years have been implicated [5]. The teeth affected also indicate a systemic cause at or around the time of birth [6], however the aetiology remains unclear. Possible causes are asthma, pneumonia, upper respiratory tract infections, otitis media, antibiotics, dioxins in mother's milk, tonsillitis, and tonsillectomy and exanthamatous fevers of childhood [6]. These lead to a disruption of ameloblastic activity during transitional and maturational stages of amelogenesis [7]. Also, maxillary first permanent molars (FPM) are more frequently affected by MIH than mandibular FPMs [3].

Jeremias et al. [8] explored the genetic carriage potentially involved in MIH development [8]. In this family-based association study it was reported that a susceptibility to develop MIH was 
associated with variations in genes related to amelogenesis, a conclusion which agreed with a multifactorial aetiology for MIH. The soft porous enamel of affected teeth when subjected to masticatory stress leads to post-eruptive breakdown of enamel, making the tooth susceptible to thermal stimuli [9]. This susceptibility can also be due to underlying pulpal inflammation $[10,11]$. In addition, children with MIH have a higher risk for dental caries than children without MIH [12]. Restorative treatment is challenging due to difficulties in achieving adequate anesthesia, and because of the poor adhesion of filling materials to hypomineralized enamel $[13,14]$. The affected molars can become carious and require complex restorations (adhesive intra-coronal restorations to extra coronal restorations e.g. preformed metal crowns) and there may also be repeated breakdown of tooth and restoration[6,9]. Restorations in hypomineralized molars appear to fail frequently; there is little evidence-based literature to facilitate clinical decisions on cavity design and material choice [5].

When FPMs are severely hypomineralized, restorations may be impossible, and extraction must be considered [15]. However, early orthodontic assessment is recommended in these cases to assess buccal segment crowding, occlusal relationships, and the condition of other erupted and unerupted teeth [16]. The ideal time for the loss of the mandibular FPM is before the eruption of the second permanent molar, usually at a chronological age of 8-9 years [17]. The second molar may erupt early and a good contact area relationship can eventually be established with the second premolar [18].

The hypotheses for this cross-sectional study are

1. There is a low prevalence of MIH in the Trinidad and Tobago

2. MIH prevalence is not equal in all ethnic groups in Trinidad and Tobago

3. The is no gender predilection with MIH in Trinidad and Tobago

This epidemiologic survey was conducted to ascertain the prevalence of MIH in Trinidad and Tobago, and to determine if there is any association with ethnicity and gender. This baseline data resources can be planned for molar incisor hypomineralization prevention because managing molar incisor hypomineralization is a challenge for paediatric dentists. This study is reported in accordance with STROBE guidelines.

\section{Methods}

The University of The West Indies ethics committee granted approval for this cross-sectional survey. The Ministry of Education in Trinidad and Tobago then gave approval to conduct this research in high schools across the country. High school Principals were then contacted to obtain permission to conduct the research. Consent forms were given out to the students in the first year of high school, where permission had been obtained from school principals. Only students from whom consent was obtained from both parent and child were examined.

\section{The study population}

One clinician (TH) examined the students who were selected from thirty out of 141 public high schools which gave permission to conduct the research. These schools were located across the twin island republic, representing both rural and urban populations between the periods January 2015 to April 2016. This epidemiological survey comprised 532 high school children aged 11 to 12 years. The children with generalized hypoplastic/ hypomineralized defects such as amelogenesis imperfecta and those suffering from any chronic illness were excluded from the study.

\section{Recording Procedure}

The students were examined at school in a well-lit area. The status of first permanent molars and all permanent incisors were evaluated with visual inspection with the use of a dental mirror. The students were seated on a chair and the molars and incisors were examined without drying to rule out opacities due to excessive drying and the size of the lesion was not taken into account. Intraoral photographs were taken. Children were considered to have MIH if one or more PFM with or without involvement of incisors met the diagnostic criteria.

\section{Statistical Analysis}

The data was coded, entered into a computer and analyzed using a statistical package (IBM SPSS Statistics for Windows, version 22 (IBM Corp., Armonk, N.Y., USA)). The data was first checked for any inclusion errors. Candidates outside the age range of 11-12 years were removed. Descriptive analysis was undertaken. The frequency of MIH was calculated for the total population. Analysis of gender and ethnic differences were also tabulated, a value of $\mathrm{p}<0.05$ was regarded as significant.

\section{Results}

In total 532 children aged 11-12 years were examined. The sample consisted of $58.1 \%$ female and $41.9 \%$ male. $12.5 \%$ were aged 11 and $86.3 \%$ were aged 12 . The mean age was 11.9 years. There were $62 \%$ Afro- Trinidadian, 22\% Indo-Trinidadian and 16\% of mixed ethnicity. Data of 532 children were analyzed to identify those diagnosed with MIH. Table 1 shows 7 out of 532 had at least one molar affected by hypomineralization which represented prevalence of $1.3 \%$. Maxillary molars were the more frequently hypomineralization (chi squared $p=0.001$ ). 6 out of 7 of the affected molars were from Afro-Trinidadians but chi-square analysis showed these differences were not statistically significant $p>0.005$ (Table 2). Looking at gender females had a higher prevalence for hypomineralization molars. 
Table 1: Total and individual prevalence of $\mathrm{MIH}$.

\begin{tabular}{|c|c|c|c|c|}
\hline \multicolumn{2}{|c|}{} & \multicolumn{2}{c|}{ Molar } & Notal \\
\hline \multirow{2}{*}{ Gender } & & MIH subject & Non-MIH subject & 303 \\
& Female & $5(71.4 \%)$ & 298 & 229 \\
\cline { 2 - 5 } & Male & $2(28.6 \%)$ & 525 & 532 \\
\hline Total & & 7 & & \\
\hline
\end{tabular}

Table 2: Association between ethnicity and $\mathrm{MIH}$.

\begin{tabular}{|c|c|c|c|c|}
\hline \multicolumn{2}{|c|}{} & \multicolumn{2}{|c|}{ Molar } & \multicolumn{2}{c|}{ Non-MIH subject } \\
\hline \multirow{3}{*}{ Ethnicity } & Afro-Trinidadian & MIH subject & 324 & 330 \\
\cline { 2 - 6 } & Indo-Trinidadian & 6 & 117 & 117 \\
\cline { 2 - 6 } & Mixed & 0 & 84 & 55 \\
\hline Total & & 7 & 525 & 532 \\
\hline
\end{tabular}

\section{Discussion}

Using G*Power [19] of determining adequacy of the sample size it was calculated that a $2 \times 2$ chi square with 1 degree of freedom and our sample of 532 achieved a power to detect a hypothetical medium effect $(\mathrm{w}=0.3)$ and found that achieved power for all chi squares will exceed $99.9 \%$. This means that in the case of null results you can argue that either there is no effect to detect, or it is very small. Paediatric patients are not routinely screened for the presence of MIH affected molars and incisors. Researchers have found MIH is a common finding reported as ranging from $2.8 \%$ to $44 \%$ prevalence [20]. Studies from Kenya reported a $13.7 \%$ prevalence [21], studies from India reported 13.9\%9, and studies from Hong Kong 2.8\% [22].

The ranges in prevalence may reflect real differences between regions and countries. However, this could also be explained partly by the different ages of children at examination, different birth cohorts, local environmental circumstances, differences in diagnostic criteria and whether hypoplasia and or opacities were included [23]. In this study a MIH prevalence of $1.3 \%$ was found. Females in this study showed a slightly higher prevalence of MIH which is in agreement with other studies [24,25]. This difference was not statistically significant for molars $(p>0.05)$. Some studies though do not report a gender related difference [26,27]. AfroTrinidadians were more likely to have PFM with MIH but the number of MIH cases was small and so chi-square test found no statistical significance between the different ethnic groups.

The prevalence in this study was low and another country with the low recorded prevalence is in Hong Kong, at 2.4\% for a similar age group [22] in a retrospective study using clinical records. At the 11 to 12-year age the molars and incisors are erupted and signs of MIH could be disturbed by caries and restorations since the teeth would have been exposed to the oral cavity long enough [23]. But the caries experience of 12-year olds in Trinidad and Tobago is reported to be a low with a DMFT of 0.61[28]. Whether extractions were due to MIH was unknow also the presence of atypical restorations and fissure sealants were not recorded so the true prevalence could have been underestimated. Severity of hypomineralization was not recorded, nevertheless this study does provide baseline data for the Trinidad and Tobago population which at the time of writing was not available in the literature.

\section{Conclusion}

a. This study reports low prevalence of $\mathrm{MIH}, 1.3 \%$ in children aged 11-12 years in Trinidad and Tobago.

b. Females were more affected with MIH

c. There is no statistically significant difference in prevalence among ethnic groups

d. Even though MIH has a low occurrence in the pediatric population in Trinidad and Tobago preventative measures for post eruptive breakdown and caries should be looked at namely in the health systems in Trinidad and Tobago.

\section{References}

1. Weerheijm KL (2004) Molar incisor hypomineralization (MIH): clinical presentation, aetiology and management. Dent Update 31(1): 9-12.

1. Calderara PC (2005) The prevalence of Molar Incisor Hypomineralisation (MIH) in a group of Italian school children. Eur J Paediatr Dent 6(2): 79-83.

2. Leppaniemi A, PL Lukinmaa, S Alaluusua (2001) Nonfluoride hypomineralizations in the permanent first molars and their impact on the treatment need. Caries Res 35(1): 36-40.

3. Beentjes VEVM WK, Groen HJ (2000) A match control study into the aetiology of hypomineralized first molar. European Academy of Paediatric Dentistry Congress. European Journal Paediatric Dentistry 1: 123. 
4. William V, LB Messer, MF Burrow (2006) Molar incisor hypomineralization: review and recommendations for clinical management. Pediatr Dent 28(3): 224-232.

5. Willmott NS, RA Bryan, MS Duggal (2008) Molar-incisor-hypomineralisation: a literature review. Eur Arch Paediatr Dent 9(4): 172-179.

6. Fearne J, P Anderson, GR Davis (2004) 3D X-ray microscopic study of the extent of variations in enamel density in first permanent molars with idiopathic enamel hypomineralisation. Br Dent J 196(10): 634638.

7. Jeremias F (2016) Family-Based Genetic Association for Molar-Incisor Hypomineralization. Caries Res 50(3): 310-318.

8. Mishra A, RK Pandey (2016) Molar Incisor Hypomineralization: An Epidemiological Study with Prevalence and Etiological Factors in Indian Pediatric Population. Int J Clin Pediatr Dent 9(2): 167-171.

9. Fagrell TG (2010) Chemical, mechanical and morphological properties of hypomineralized enamel of permanent first molars. Acta Odontol Scand 68(4): 215-222.

10. Rodd HD (2007) Pulpal expression of TRPV1 in molar incisor hypomineralisation. Eur Arch Paediatr Dent 8(4): 184-188.

11. Wuollet E (2018) The Association between Molar-Incisor Hypomineralization and Dental Caries with Socioeconomic Status as an Explanatory Variable in a Group of Finnish Children. Int J Environ Res Public Health 15(7): 1324.

12. Kotsanos N, EG Kaklamanos, K Arapostathis (2005) Treatment management of first permanent molars in children with Molar-Incisor Hypomineralisation. Eur J Paediatr Dent 6(4): 179-184

13. Lygidakis NA (2010) Best Clinical Practice Guidance for clinicians dealing with children presenting with Molar-Incisor-Hypomineralisation (MIH): An EAPD Policy Document. Eur Arch Paediatr Dent 11(2): 7581.

14. Weerheijm KL (2001) Prevalence of cheese molars in eleven-year-old Dutch children. ASDC J Dent Child 68(4): 259-262.

15. Fayle SA (2003) Molar incisor hypomineralisation: restorative management. Eur J Paediatr Dent 4(3): 121-126.
16. Thunold K (1970) Early loss of the first molars 25 years after. Rep Congr Eur Orthod Soc pp. 349-365.

17. Gill DS, RT Lee, CJ Tredwin (2001) Treatment planning for the loss of first permanent molars. Dent Update 28(6): 304-308.

18. Faul F (2007) G*Power 3: a flexible statistical power analysis program for the social, behavioral, and biomedical sciences. Behav Res Methods 39(2): 175-191.

19. Jalevik B (2010) Prevalence and Diagnosis of Molar-Incisor- Hypomineralisation (MIH): A systematic review. Eur Arch Paediatr Dent 11(2): 59-64.

20. Kemoli AM (2008) Prevalence of molar incisor hypomineralisation in six to eightyear-olds in two rural divisions in Kenya. East Afr Med J 85(10): 514-519.

21. Cho SY, Y Ki, V Chu (2008) Molar incisor hypomineralization in Hong Kong Chinese children. Int J Paediatr Dent 18(5): 348-352.

22. Weerheijm KL, I Mejare (2003) Molar incisor hypomineralization: a questionnaire inventory of its occurrence in member countries of the European Academy of Paediatric Dentistry (EAPD). Int J Paediatr Dent 13(6): 411-416.

23. Aslam RNK, S Rizwan (2014) Frequency of malocclusion among 12-15 years old school children in three sectors of Karachi. Pakistan Oral \& Dental Journal 34(3): 510-514

24. Lygidakis NA, G Dimou, D Marinou (2008) Molar-incisor-hypomineralisation (MIH). A retrospective clinical study in Greek children. II. Possible medical aetiological factors. Eur Arch Paediatr Dent 9(4): 207-217.

25. Allazzam SM, SM Alaki, OA El Meligy (2014) Molar incisor hypomineralization, prevalence, and etiology. Int J Dent 2014. 234508.

26. Garcia Margarit M (2014) Epidemiologic study of molar-incisor hypomineralization in 8-year-old Spanish children. Int J Paediatr Dent 24(1): 14-22.

27. Naidu R, I Prevatt, D Simeon (2006) the oral health and treatment needs of schoolchildren in Trinidad and Tobago: findings of a national survey. International journal of paediatric dentistry 16(6): 412-418. 